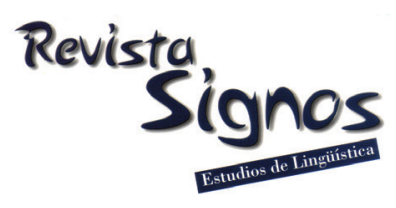

\title{
El resumen del artículo de investigación: Análisis del género en un corpus de textos de Enfermería
}

\author{
The research article abstract: \\ Genre analysis in a Nursing corpus of texts
}

\author{
Carmen Piqué-Noguera \\ UNIVERSITAT DE VALÈNCIA \\ ESPAÑA \\ carmen.pique@uv.es
}

\author{
Ramón Camaño-Puig \\ Universitat de VALÈnCIA \\ EsPaña \\ ramón.camano@uv.es
}

Recibido: 17-10-2012 / Aceptado: 11-VI-2014

\section{Resumen}

El resumen es un género que ha recibido un tratamiento desigual en la literatura especializada; mientras en biomedicina, ciencias sociales y otras disciplinas afines numerosos autores han estudiado el resumen (Salager-Meyer, 1990; Hyland, 2000; Swales \& Feak, 2009); en otras, en cambio, se les ha prestado escasa atención en lo que a su estructura y contenido se refiere y, concretamente, en Enfermería. Su importancia en la diseminación de la ciencia debería ser un acicate para dotar el resumen de una mayor información y fuerza persuasiva. Lo poco que se ha escrito sobre el resumen no aborda la problemática de si el resumen debe seguir el modelo tradicional de un solo párrafo o el estructurado con subtítulos (Posteguillo Gómez \& Piqué-Angordans, 2007). El objetivo del presente estudio es describir el contenido y estructura del resumen tradicional así como los elementos textuales y discursivos del mismo. Siguiendo el modelo de la literatura especializada (Weissberg \& Buker, 1990), se ha realizado un análisis de un corpus de 240 resúmenes extraídos de artículos de doce revistas de Enfermería mediante la identificación de sus unidades estructurales. Entre los resultados más significativos destaca la amplia variabilidad en cuanto a la estructura y contenido del resumen, con un exceso de resúmenes indicativos más que informativos. A partir de estos resultados concluimos que hace falta unificar criterios para una mejor redacción de los resúmenes de Enfermería y promoción de la investigación, con contenidos más afines a las directrices de la crítica especializada.

Palabras Clave: Resumen del artículo de investigación, estudio del género, resumen tradicional y estructurado ciencias de la salud, Enfermería. 


\begin{abstract}
The abstract is a genre that has received a great deal of attention in some areas, such as biomedicine and the social sciences (Salager-Meyer, 1990; Hyland, 2000; Swales \& Feak, 2009); however, its structure and content in other areas, such as nursing, has received less attention. Its importance in the dissemination of science should be an incentive for nursing authors to make it more informative and persuasive. No research has been found that surveyed the issue of whether the abstract should follow the traditional model of a single paragraph or structured with subtitles (Posteguillo Gomez \& PiquéAngordans, 2007). The aim of this study is to describe the content and structure of the traditional abstract, as well as its textual and discursive features. Drawing on the work of Weissberg and Buker (1990), we conducted an analysis of a corpus of 240 abstracts retrieved from twelve nursing journals in which we identified their structural units. One the most striking results was the wide range of variability in terms of the structure and content of the abstracts, with a vast majority of indicative, rather than informative abstracts. These findings suggest the need for a set of agreed upon criteria for writing abstracts in the field of nursing, more clearly aligned with international guidelines for scientific publication.
\end{abstract}

Key Words: Research article abstract, genre study, traditional vs. structured abstracts, health sciences, Nursing.

\title{
INTRODUCCIÓN
}

El artículo de investigación ha sido extensamente estudiado por la lingüística aplicada. Swales (1990), uno de los pioneros en la investigación del artículo, ha mantenido que el resumen, como parte integrante del mismo, es uno de los géneros olvidados por los analistas del discurso. El panorama, sin embargo, ha cambiado radicalmente en los últimos veinte años, sobre todo en áreas como la Medicina (Salager-Meyer, 1990; Hyland, 2000; Nakayama, Hirai, Yamazaki \& Naito, 2005; Swales \& Feak, 2009), las Ciencias Sociales (Hartley, 2002; Martín Martín, 2003), en los negocios (Piqué-Noguera, 2012), y en trabajos multidisciplinares (Hyland, 2000; Fortanet Gómez, 2002). A excepción de la Medicina, en ciencias de la salud en general y concretamente en Enfermería los trabajos sobre el artículo de investigación son escasos (Newell, 2000; Piqué-Angordans, Camaño-Puig \& Piqué-Noguera, 2009, 2011) y mucho más escasa es la presencia de artículos sobre el resumen en particular, quizá por no ser considerado un género importante, pese a ser fundamental en la diseminación de la ciencia (Díez, 2007).

La literatura de Enfermería apenas ha cubierto el tema sobre la importancia de publicar resúmenes de tipo no estructurado o tradicional (en lo sucesivo) o estructurados, ni tampoco cómo redactarlos. Tanto las revistas de Enfermería como los estudios en lingüística aplicada no han abordado el tema de si vale la pena unificar criterios en esta área. Pensamos que los consejos editoriales de las revistas de Enfermería analizadas, que se vienen caracterizando por su indefinición, deben 
dar un paso hacia adelante y pronunciarse sobre cuál creen que es la función de este género y cómo debe redactarse. Piqué-Angordans et al. (2009) señalan que muchas revistas de Enfermería publican según las directrices de las revistas médicas y el modelo Vancouver, pero es preciso aclarar y unificar criterios, especialmente con respecto al resumen, por lo que este debate, sobre si el resumen ha de ser estructurado o no, sigue vigente. En varias disciplinas también se está debatiendo el tema. Por ejemplo, Hartley, en Ciencias Sociales, opina que es aconsejable utilizar resúmenes estructurados ya que son más informativos y porque "transmiten su estructura interna más claramente que los tradicionales” (Hartley, 1997: 316); de igual forma lo hacen Mosteller, Nave y Miech (2004), en Educación, aunque difieren de Hartley (1997) en que este aboga por cinco sub-apartados reflejo de la estructura del artículo (IMRD) ${ }^{1}$, mientras que Mosteller et al. (2004) prefieren nueve, a semejanza del modelo médico actual.

Según Piqué-Angordans et al. (2011: 113), el resumen:

"significa cosas distintas a autores distintos, pero pensamos que todos deberían estar de acuerdo en que el resumen [...] debería reflejar en cierto modo la estructura del artículo mismo".

Otañi (2005: 48) destaca el "rol anticipador" del resumen pues se trata de "una anticipación del contenido y de la estructura del texto". Sin embargo, no debe entenderse el resumen como una réplica resumida del artículo, sino como una herramienta retórica "para persuadir a los lectores de que vale la pena leer el artículo" (Hyland, 2000: 64). La avalancha actual de publicaciones, tanto en papel como en forma digital, hace inviable su control y lectura por parte del investigador o del profesional, a no ser que disponga de una herramienta que le ayude a poder distinguir las publicaciones que le puedan interesar. De ahí que la importancia del resumen haya crecido exponencialmente en los últimos años. Por esta razón es importante dotar el resumen de la información adecuada para que se convierta realmente en el elemento de marketisation del artículo científico, según el propio Hyland (2000).

Como ha comentado Hyland (2000), el resumen es un género clave para asentar una disciplina y para que sea reconocida como tal por los lectores en general y especialistas en particular. La teoría del análisis del género es un campo de estudio muy prometedor con un considerable contenido para potenciar la investigación en el área de la comunicación en Enfermería. Aunque el resumen ha sido relativamente ignorado por la crítica (Swales, 1990), Hyland (2000: 83), en su estudio multidisciplinar del resumen como género, comenta que si está bien escrito ofrece "una visión fascinante de la práctica discursiva de comunidades académicas diferentes".

En su análisis sobre el avance del conocimiento en los primeros años de la actividad académica, Shapin (1984: 481) sugería que "generar conocimiento y su comunicación son acciones igualmente importantes", de ahí lo primordial que era “crear también un público científico". Esto se consigue a través del género como 
acción social, de la escritura, de las publicaciones y de su lectura, todo lo cual forma parte de esta producción de conocimiento. Bazerman (1988: 10) lo resumía con las siguientes palabras:

"escribir es una acción social; los textos ayudan a organizar actividades sociales y estructura social; y la lectura es una forma de participación social; por lo tanto, decir algo sobre la escritura es decir algo sobre la sociología”.

\section{Resumen tradicional versus estructurado}

En la literatura especializada sobre el resumen han surgido dos temas recurrentes: por una parte, la dicotomía entre resumen indicativo y resumen informativo, y por otra, la elección entre el resumen tradicional o el estructurado. El resumen indicativo "tiene por objeto indicar el tema del artículo" (Day, 2005: 29) mediante los antecedentes de la investigación y el objetivo del trabajo; ocasionalmente aparece también algún dato relacionado con la metodología o con los resultados, lo que da lugar a un tercer tipo de resumen, el indicativo-informativo. Por otro lado, en el resumen informativo, según la National Information Standards Organization (ANSI/NISO, 1997) y la mayoría de los manuales de estilo, como el de la American Psychological Association (APA, 2010), por lo general se incluye información sobre antecedentes, objetivo, metodología, resultados y conclusión, en cierto modo siguiendo la estructura del artículo científico al que representa. La crítica reciente, no obstante, apenas hace mención de esta clasificación.

El segundo tema recurrente entre los especialistas ha sido el debate entre el resumen tradicional y el resumen estructurado. El resumen tradicional consiste en un único párrafo con cinco unidades estructurales, mientras que en el resumen estructurado estas unidades vienen delimitadas por subtítulos. La literatura suele convenir en que los resúmenes estructurados son más extensos y, en consecuencia, conllevan más información que los resúmenes tradicionales (Hartley, 2002). El presente análisis se centra en los resúmenes tradicionales a partir de los modelos de Weissberg y Buker (1990) y de Hyland (2000). En la Tabla 1, se presenta el primero de ellos con la nomenclatura y descripción de los elementos que lo componen: 
Tabla 1. Unidades estructurales (AOMRC) del resumen tradicional (traducido de

Weissberg \& Buker, 1990: 186; cursiva de los autores).

\begin{tabular}{|ll|l|}
\hline \multicolumn{2}{|l|}{ Unidad estructural } & Descripción \\
\hline $\mathbf{A}$ & $=$ & información sobre los antecedentes del tema \\
$\mathbf{O}$ & $=$ & objetivo o actividad principal del estudio y su ámbito \\
$\mathbf{M}$ & $=$ & información sobre la metodología empleada en el estudio \\
$\mathbf{R}$ & $=$ & resultados más importantes del estudio \\
$\mathbf{C}$ & $=$ & frase de conclusión o recomendación \\
\hline
\end{tabular}

\section{Objetivo}

La carencia de estudios sobre resúmenes en el área de Enfermería constituye la principal motivación para esta investigación. Pensamos que, al igual que Hartley (1997, 2002), la Enfermería también se distancia de la estructura de hasta siete subapartados que proponen en Medicina (Ad Hoc Working Group, 1987) y plantea un resumen de cinco. Por sus características, la Enfermería, que a menudo tiene poco que ver con la Biomedicina y mucho con las Ciencias Sociales, podría proponer una estructura semejante a los cinco sub-apartados sugeridos por Hartley (1997), que es la misma propuesta, con subtítulos, que la de Weissberg y Buker (1990) o la de Hyland (2000). Por lo tanto, como objetivo principal nos planteamos describir el resumen tradicional tanto en su contenido y estructura como en los elementos textuales a través del análisis de un corpus de 240 resúmenes extraídos de 12 revistas de Enfermería: nos basamos, tanto en su contenido como en su tamaño y estructura; es decir, si la información incluida es representativa de los artículos a los que preceden, e incluso si la información es consecuencia directa del tamaño de los mismos. Y, por consiguiente, si los resúmenes deben seguir el modelo tradicional o un modelo estructurado con subtítulos.

\section{Descripción del corpus y método de análisis}

\subsection{Selección de textos y estadística básica}

Nuestro corpus se compone de 240 resúmenes de tipo tradicional publicados en castellano; 20 resúmenes procedentes de cada una de las doce revistas siguientes (con indicación de la abreviatura que emplearemos y de los años a que corresponden los resúmenes del corpus): 
Tabla 2. Revistas de Enfermería que componen el corpus de resúmenes.

\begin{tabular}{|l|l|l|l|}
\hline & Revista & Abreviatura & Años \\
\hline 1. & Cultura de los Cuidados & Cult Cuidados & $2008-2011$ \\
2. & Enfermería Clínica & Enferm Clínica & $2008-2011$ \\
3. & Enfermería Comunitaria & Enferm Comunit & $2006-2011$ \\
4. & Enfermería Docente & Enferm Docente & $2008-2011$ \\
5. & Enfermería Global & Enferm Global & 2010 \\
6. & Rev. Soc. Esp. de Enfermería Nefrológica & Enferm Nefrol & $2010-2011$ \\
7. & Rev. Asoc. Esp. Enfermería en Urología & Enfuro & $2008-2010$ \\
8. & Evidentia & Evidentia & $2007-2011$ \\
9. & Gerokomos & Gerokomos & $2009-2011$ \\
10. & Index de Enfermería & Index Enferm & $2009-2011$ \\
11. & Inquietudes & Inquietudes & $2003-2011$ \\
12. & Metas de Enfermería & Metas Enferm & $2010-2011$ \\
\hline
\end{tabular}

Las revistas mencionadas en la Tabla 2 fueron elegidas en función de su aparición en el listado de revistas en el Índice de Repercusión Histórica de la Fundación Índex de Enfermería, así como de su disponibilidad a través de la Universitat de València. Inicialmente optamos por centrar nuestra investigación en los resúmenes publicados en el año 2010, por ejemplo, 'Enferm Global 'y 'Enfuro'2; sin embargo, la poca disponibilidad de resúmenes de tipo tradicional en algunas de ellas nos obligó a extender nuestra búsqueda hasta localizar 20 resúmenes por revista. Exceptuando 'Enferm Global', en las revistas analizadas se vienen publicando artículos con ambos tipos de resúmenes.

Una vez seleccionado, se etiquetó el corpus para la contabilización del número de palabras por frase y por párrafo (o resumen), su desviación estándar (DE), y para la extracción de concordancias a través del programa WordSmith Tools (Scott, 2009). Dado que partimos del concepto tradicional de resumen de un solo párrafo (Weissberg \& Buker, 1990; Day, 2005; APA, 2010), hemos considerado los resúmenes que vienen formateados en diversos párrafos como de uno solo para su análisis.

A pesar de lo reducido de la muestra, pensamos que se pueden extraer datos importantes para una mejor redacción del resumen. En la Tabla 3 presentamos los datos numéricos básicos correspondientes a las doce revistas de Enfermería analizadas, con expresión del número de palabras que suman los resúmenes de cada revista, juntamente con el promedio de palabras por resumen y su desviación estándar (DE): 
Tabla 3. Estadística básica del corpus de resúmenes.

\begin{tabular}{|l|c|c|c|}
\hline & $\begin{array}{c}\text { Número de palabras de los 20 } \\
\text { resúmenes de cada revista }\end{array}$ & $\begin{array}{c}\text { Palabras por } \\
\text { resumen }\end{array}$ & DE \\
\hline Cult Cuidados & 3.336 & 166,80 & 57,87 \\
\hline Enferm Clínica & 3.611 & 180,55 & 46,22 \\
\hline Enferm Comunit & 3.078 & 153,90 & 41,73 \\
\hline Enferm Docente & 2.675 & 133,75 & 36,67 \\
\hline Enferm Global & 3.787 & 189,35 & 46,84 \\
\hline Enferm Nefrol & 4.710 & 235,50 & 43,46 \\
\hline Enfuro & 2.641 & 132,05 & 55,28 \\
\hline Evidentia & 3.396 & 169,80 & 42,19 \\
\hline Gerokomos & 3.228 & 161,40 & 49,63 \\
\hline Index Enferm & 2.721 & 136,05 & 30,15 \\
\hline Inquietudes & 2.688 & 134,40 & 51,66 \\
\hline Metas Enferm & 3.541 & 177,05 & 56,79 \\
\hline
\end{tabular}

Cabe destacar la alta DE en la mayoría de revistas, indicativo de su variabilidad en extensión, como en 'Cult Cuidados', con un mínimo y máximo de palabras de 82 y 298, con lo cual se dispara la DE a la cifra de 57,87. Lo mismo ocurre en 'Enfuro', con 57 y 247 palabras y una DE de 55,28. En cambio, 'Enferm Nefrol' es la única revista en la que todos sus resúmenes superan las 150 palabras, por lo que su DE $(43,46)$ es bastante inferior. Asimismo, 'Index Enferm' ofrece una mayor regularidad en sus resúmenes, con un promedio de 136,06 palabras por resumen y con la DE más baja $(30,15)$ de las revistas estudiadas.

\subsection{Método de estudio}

Para analizar la estructura de un género como es el artículo de investigación, la lingüística anglosajona utiliza términos como move y step, es decir, 'movimiento' y 'paso', al objeto de distinguir las diversas funciones y sub-funciones comunicativas de un artículo de investigación (Swales, 1990). En la obra colectiva de Fortanet Gómez (2002) se optó por 'secuencia' y 'unidad informativa', por lo que en el capítulo de esa obra sobre el resumen se habla de una estructura basada en 'secuencias' (Posteguillo Gómez, 2002). En el presente trabajo, no obstante, hemos optado por 'unidad estructural' siguiendo la investigación de Martín Martín (2003), entendiéndola como una unidad de análisis "para describir las estructuras discursivas de un género específico” (Martín Martín, 2010: 114).

En la presente investigación, por lo tanto, entendemos por 'unidad estructural' un segmento de un texto que realiza una función comunicativa específica (Swales, 1990). Cada unidad no solo tiene su propio objetivo (Tabla 1), sino que también contribuye a los objetivos comunicativos generales del género. El análisis de las unidades estructurales no se basa en criterios únicamente lingüísticos (enfoque bottom-up), sino también y por separado en criterios de contenido (enfoque top-down); es decir, "una vez 
identificadas las unidades estructurales, se investigan las características lingüísticas típicas de cada unidad" (Pho, 2008: 233); con estos parámetros se delimitan las unidades que conforman y delimitan la estructura interna del resumen.

La obtención de estos datos se ha basado en la discriminación manual entre una unidad y otra de cada resumen, tarea que no siempre ha sido fácil, debido al solapamiento de unas con otras. En otras palabras, se ha buscado diferenciar la intención comunicativa del autor mediante el uso de estrategias retóricas determinadas. En los resúmenes analizados, hay claros ejemplos de cada una de ellas. Por ejemplo, en 'Antecedentes' con frecuencia se hace referencia a la bibliografía previa con el uso del pretérito perfecto compuesto (nuestro subrayado), como en el siguiente ejemplo (1):

(1) Se han estudiado y desarrollado mejoras en la técnica de punción [...] (Vizmanos Sevilla, Alonso Ugartemendía, Arenas Olmo, Montilla Martín, Ruiz Serrato \& Grupo de Cuidados Obstétricos, 2010).

Con aún más claridad aparece la unidad de 'Objetivo' con expresiones típicas, como "Se pretende averiguar [...]", "El objetivo de esta investigación [...]", o variantes como en el ejemplo (2):

(2) El presente estudio tiene como objetivo describir el problema de la soledad en el anciano [...] (Rodríguez Martín, 2009)

En 'Metodología', no obstante, la diferenciación a veces presenta mayores dificultades, especialmente cuando no se trata de un artículo basado en datos empíricos, aparte de que con frecuencia se presenta en combinación con otra unidad estructural. Véase el ejemplo (3), en el que se combinan las unidades 'Objetivo' [O] y 'Metodología' [M]:

(3) $[\mathrm{O}]$ El objetivo del presente estudio es evaluar la eficacia de la infusión automática comparada con la manual en la HDF on-line post-dilucional, $[\mathrm{M}]$ mediante la medición del volumen convectivo final y del aclaramiento de pequeñas moléculas mediante el Kt. (Fernández Martínez, Soto Ureña, Arenas Fuentes, Horrillo Jiménez, Sáez Donaire \& Pérez Valencia, 2010)

El ejemplo (4) ilustra claramente un caso de la unidad 'Resultados', aunque esta unidad suele aparecer con un sinfín de fórmulas para expresarla:

(4) Los resultados muestran diferencias discretas, algunas significativa, pero todas en la dirección de [...] (Perea Baena, 2008)

La unidad 'Conclusión' también ofrece diversas posibilidades para diferenciarla de 'Resultados', ya que una frase puede ser interpretada como perteneciente a una u otra unidad, como podría suceder con el ejemplo (5). Sin embargo, a la vista del 
resumen en su totalidad, se deduce que se refiere a 'Conclusión' (el verbo 'sugerir' tiene a menudo esta doble función, todo lo contrario que con el verbo 'concluir', ejemplo (6)):

(5) La observación sugiere la existencia de una relación funcional pero un tanto distante entre los empleados de la institución y los pacientes. (Kandzior, 2009)

(6) Se puede concluir que los diagnósticos enfermeros no se han modificado sustancialmente ni en presencia ni en frecuencia [...] (Rodríguez Borrego, Galván Ledesma, Medina Valverde, de la Torre Barbero, Romero Bravo \& Flórez Almonacid, 2010)

\section{Análisis de resultados}

Como primer paso en el análisis de los resultados, siguiendo el método de estudio para la identificación de las distintas unidades estructurales, se presenta en la Tabla 4 la descripción detallada por revista y unidad estructural del contenido de los resúmenes del corpus:

Tabla 4. Total de unidades estructurales por revista (porcentajes entre paréntesis).

\begin{tabular}{|c|c|c|c|c|c|c|}
\hline & A & O & $\mathbf{M}$ & $\mathbf{R}$ & $\mathrm{C}$ & Promedio \\
\hline Cult Cuidados & $17(85 \%)$ & $20(100 \%)$ & $11(55 \%)$ & $8(40 \%)$ & $5(25 \%)$ & $12,2(61,0 \%)$ \\
\hline Enferm Clínica & $17(85 \%)$ & $16(80 \%)$ & $6(30 \%)$ & $2(10 \%)$ & 0 & \\
\hline Enferm Comunit & $15(75 \%)$ & $14(70 \%)$ & $14(70 \%)$ & $11(55 \%)$ & $5(25 \%)$ & 11,8 \\
\hline Enferm Docente & $16(80 \%)$ & $18(90 \%)$ & $6(30 \%)$ & $5(25 \%)$ & $2(10 \%)$ & $9,4(47,0 \%)$ \\
\hline Enferm Global & $9(45 \%)$ & $19(95 \%)$ & $17(85 \%)$ & $17(85 \%)$ & $5(25 \%)$ & 13,4 \\
\hline Enferm Nefrol & $16(80 \%)$ & $20(100 \%)$ & $18(90 \%)$ & $18(90 \%)$ & $10(50 \%)$ & 16,4 \\
\hline Enfuro & $11(55 \%)$ & $20(100 \%)$ & $14(70 \%)$ & $8(40 \%)$ & $14(70 \%)$ & 13, \\
\hline Evidentia & $17(85 \%)$ & $19(95 \%)$ & $10(50 \%)$ & $12(60 \%)$ & $4(20 \%)$ & $2,0 \%)$ \\
\hline Gerokomos & $13(65 \%)$ & $16(80 \%)$ & $12(60 \%)$ & $12(60 \%)$ & $3(15 \%)$ & $11,2(56,0 \%)$ \\
\hline Index Enferm & $16(80 \%)$ & $19(95 \%)$ & $10(50 \%)$ & $9(45 \%)$ & $4(20 \%)$ & $8,0 \%)$ \\
\hline Inquietudes & $14(70 \%)$ & $19(95 \%)$ & $8(40 \%)$ & $5(25 \%)$ & $5(25 \%)$ & $10,2(51,0 \%)$ \\
\hline & $17(85 \%)$ & $16(80 \%)$ & $8(40 \%)$ & $5(25 \%)$ & $2(10 \%)$ & $9,6(48,0 \%)$ \\
\hline Totales & $\begin{array}{r}14,8 \\
(74,2 \%) \\
\end{array}$ & $\begin{array}{r}18,0 \\
(90,0 \%)\end{array}$ & $\begin{array}{r}11,2 \\
(55,8 \%) \\
\end{array}$ & $\begin{array}{r}9,3 \\
(46,6 \%) \\
\end{array}$ & $\begin{array}{r}4,9 \\
(24,6 \%) \\
\end{array}$ & $\begin{array}{r}11,7 \\
(58,3 \%)\end{array}$ \\
\hline
\end{tabular}

A - Antecedentes; O - Objetivo; M - Metodología; $\mathrm{R}$ - Resultados; C - Conclusión

En nuestros resultados, 'Enferm Nefrol' aparece como la más completa con el $82 \%$ de unidades, con porcentajes por unidad superiores al 60\%, excepto en Conclusiones, resultados que son comprensibles dado el tipo de artículos de esta revista; cuatro superan el 60\% ('Cult Cuidados', 'Enferm Global', 'Enfuro' y 'Evidentia'), otras cuatro están por encima del 50\% ('Enferm Comunit,' 'Gerokomos', 'Index Enferm’ e 'Inquietudes'), mientras que el resto no llegan a ese porcentaje, con 'Enferm Clínica' como la menos informativa con un $41 \%$. 
Globalmente, el porcentaje de unidades estructurales en nuestro corpus es del $58,3 \%$, inferior a los resultados de Martín Martín (2003) quien obtuvo un cómputo global de $73,75 \%$, en resúmenes de Psicología y Fonética, aunque muy cercano a la investigación multidisciplinar de Hyland (2000) con un porcentaje global de 58,8.

Analizando las revistas individualmente y por unidades, existe una precariedad manifiesta en la unidad de 'Resultados' (solo cuatro revistas superan el 60\%), no se pueden considerar sus resúmenes suficientemente persuasivos para que el lector adquiera y lea el artículo. En un posible ranking de presencias de unidades, se da más preponderancia a 'Antecedentes', con un promedio del 74,2\%, que a 'Resultados', con un 46,6\%. En consecuencia, solo en 'Enferm Nefrol' y 'Enferm Global' las tres unidades centrales llevan el peso de la información, que coincide con la conclusión de Hyland (2000) en su investigación multidisciplinar; en cambio 'Enfuro' baja mucho en 'Resultados', con un 40\%. No obstante, la más regular es 'Enferm Nefrol' en la que predominan los resúmenes de 4 y 5 unidades estructurales con un promedio de $82 \%$. En la unidad estructural 'Conclusión', sin embargo, los porcentajes son muy inferiores y solo una revista está por encima del 60\% ('Enfuro'), mientras que 'Enferm Clínica' omite totalmente esta unidad.

En cuanto a qué unidades estructurales son las más frecuentes, también es 'Enferm Nefrol' la que contiene el porcentaje más alto en las tres unidades centrales ('Objetivo', 100\%; 'Metodología', 90\%, y 'Resultados', 90\%), debido a que la mayoría de los resúmenes de esta revista son de tipo tradicional procedentes de artículos basados en datos empíricos. Por otro lado, 'Enferm Clínica', con unos porcentajes muy inferiores (80\%, 30\% y 10\%, respectivamente), contiene resúmenes extraídos entre 2006 y 2011. En estos seis años, la mayoría de artículos de esta revista, especialmente los llamados 'originales' y basados en datos empíricos, van precedidos de resúmenes estructurados, mientras que los de resúmenes de tipo tradicional acompañan a artículos de contenido más informativo y educativo, lo que explica en cierto modo los bajos porcentajes de 'Enferm Clínica'. Por este motivo, en un hipotético ranking de revistas, las dos últimas serían 'Metas Enferm' y 'Enferm Clínica', con un promedio de solo 10 y 8 unidades estructurales, respectivamente, en los 20 resúmenes analizados de cada revista. El Gráfico 1 muestra los resultados obtenidos en nuestro análisis: 


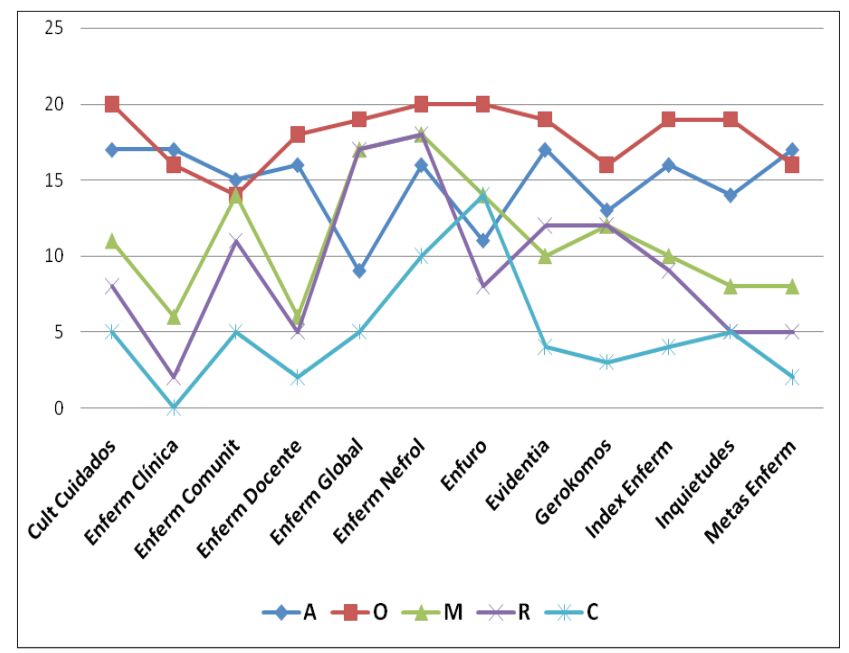

Gráfico 1. Representación visual de la presencia de unidades estructurales en los resúmenes.

Adicionalmente, hemos analizado, utilizando el paquete estadístico IBM SPSS 20, las muestras de resúmenes de cada una de las revistas para ver en qué condiciones se debería llevar a cabo el análisis estadístico. Iniciamos el proceso con la prueba estadística de Kolmogorov-Smirnov con el fin de aplicarla a cada una de las muestras de las revistas lo que dio como resultado una $\mathrm{p}>0,05$ en todos los casos. Determinada la normalidad de la distribución, se llevó a cabo la aplicación de la prueba no paramétrica de Kruskall-Wallis, obteniéndose una $\mathrm{p}>0,05$ en todos los casos, excepto en la revista 'Enferm Clínica' en la que se obtuvo una $\mathrm{p}<0,05$ en Antecedentes $(\mathrm{p}=0,008)$ y Resultados $(\mathrm{p}=0,012)$, así como en la revista 'Cult Cuidados', donde obtuvimos una $\mathrm{p}=0,020$ en la Metodología. Estos datos indican que no existen diferencias significativas entre los resúmenes de las revistas, salvo en los casos mencionados, a pesar de que se observa una alta variabilidad a la hora de escribir el resumen de un artículo de Enfermería.

Siguiendo el modelo propuesto por Weissberg y Buker (1990), en los 240 resúmenes estudiados deberían aparecer, como número ideal, 1.200 unidades estructurales, aunque su presencia es bastante escasa, como se refleja en la Tabla 5:

Tabla 5. Presencia (número total y porcentaje) de unidades en los 240 resúmenes.

\begin{tabular}{|l|c|c|}
\hline Unidad estructural & $\mathbf{n}$ & $\mathbf{\%}$ \\
\hline Antecedentes [A] & 178 & 74,17 \\
Objetivo [O] & 216 & 90,00 \\
Metodología [M] & 134 & 55,83 \\
Resultados [R] & 112 & 46,67 \\
Conclusión [C] & 59 & 24,58 \\
\hline
\end{tabular}


Estos datos apuntan hacia unos resúmenes en los que predominan 'Antecedentes' $(74,17 \%)$ y 'Objetivo' (90\%), resultados que no concuerdan con la literatura sobre las tres unidades centrales ('Objetivo', 'Metodología' y 'Resultados'), básicas en un resumen tradicional. De hecho, Cross y Oppenheim (2005) asignan porcentajes superiores a estas tres unidades en artículos científicos: 'Objetivo', 66,67\%; 'Metodología', 100\%; y 'Resultados', 100\%. Nuestros datos confirman la variabilidad en los resúmenes de Enfermería, así como la abundante presencia de altos porcentajes en 'Antecedentes' en 'Objetivo', muy superiores a 'Resultados' y 'Conclusión'.

Según la crítica especializada, las tres unidades estructurales centrales (OMR) de los resúmenes son básicas; por ejemplo, Martín Martín (2003), en resúmenes en inglés y español de Psicología, observó que los porcentajes en todas las unidades de los resúmenes en inglés superaban el 80\%; en cambio, en los textos en español, informó de "una fuerte tendencia a omitir la unidad Resultados" (Martín Martín, 2003: 166) con un porcentaje del 41,25\%. Los porcentajes de la Tabla 4 difieren significativamente, lo cual indica que muchos de los resúmenes estudiados, precisamente por su bajo porcentaje en la unidad de Resultados (46,67\%), solo indican el objetivo del artículo y el tema central del mismo.

Otro parámetro indicativo de esta carencia de información es el número de unidades estructurales que contienen los resúmenes para ser considerados más o menos completos. La Tabla 6 presenta en detalle este dato:

Tabla 6. Número de unidades estructurales en los 240 resúmenes.

\begin{tabular}{|l|c|c|}
\hline $\mathbf{N}^{\mathbf{o}}$ unidades & $\mathbf{n}$ & $\mathbf{\%}$ \\
\hline 5 unidades & 16 & 6,67 \\
4 unidades & 67 & 27,92 \\
3 unidades & 63 & 26,25 \\
2 unidades & 68 & 28,33 \\
1 unidad & 26 & 10,83 \\
\hline
\end{tabular}

Cabe destacar que solo 16 de los 240 resúmenes contienen 5 unidades estructurales. Sería interesante investigar la tendencia a omitir algunas de ellas, sobre todo en los resúmenes correspondientes a artículos experimentales. La mayoría $(82,50 \%)$ se centra en 2, 3 y 4 unidades, aunque de estos datos no se deriva que las unidades predominantes sean 'Objetivo', 'Método' o 'Resultados', que la crítica considera como importantes en los resúmenes. Por ejemplo, en Ciencias Sociales Hartley y Betts (2009) señalaron estas tres unidades como fundamentales con los siguientes porcentajes: Objetivo, $79 \%$, y en Método y Resultados, $86 \%$ cada una, mientras que solo $57 \%$ tanto en Antecedentes como en Conclusiones.

Otro dato significativo es la posible relación entre el tamaño del resumen en número de palabras y la información que contiene, que se manifiesta y contabiliza a través de sus unidades estructurales. Objetivamente, en los resúmenes debería existir 
alguna relación entre las unidades presentes y el tamaño de los mismos, pero los resultados no confirman este dato. Efectivamente, según se pudo ver en las Tablas 3 y 5, las revistas con un promedio mayor de palabras por resumen no coincidían necesariamente con las de mayor proporción de unidades, como se puede observar en la Tabla 7, distribuidas en orden decreciente de número de palabras por resumen:

Tabla 7. Relación entre promedio de palabras por resumen y porcentaje de unidades estructurales presentes en cada revista.

\begin{tabular}{|l|c|c|}
\hline Revista & $\begin{array}{c}\text { Palabras por } \\
\text { resumen }\end{array}$ & $\begin{array}{c}\text { \% de } \\
\text { unidades }\end{array}$ \\
\hline Enferm Nefrol & 235,50 & 82 \\
Enferm Global & 189,35 & 67 \\
Enferm Clínica & 180,55 & 41 \\
Metas Enferm & 177,05 & 48 \\
Evidentia & 169,80 & 62 \\
Cult Cuidados & 166,80 & 61 \\
Gerokomos & 161,40 & 56 \\
Enferm Comunit & 153,90 & 59 \\
Index Enferm & 136,05 & 58 \\
Inquietudes & 134,40 & 51 \\
Enferm Docente & 133,75 & 47 \\
Enfuro & 132,05 & 67 \\
\hline
\end{tabular}

Mientras que en la mayoría de las revistas el promedio de palabras por resumen es equiparable al porcentaje de unidades, dos de ellas, en cambio, no responden a este mismo patrón: 'Enferm Clínica' y 'Metas Enferm', con 180,55 y 177,05 palabras por resumen, respectivamente, solo tienen un $41 \%$ y $48 \%$ en concepto de unidades estructurales. Mayor es la disparidad en la revista 'Enfuro' que, con el menor número de palabras por resumen $(132,05)$, es, sin embargo, la segunda en porcentaje de información (67\%). Esta última revista, aparentemente, materializa en términos formales lo que nos preguntábamos acerca de si es posible escribir un resumen con un promedio de 150 palabras y contener la información necesaria para conducir al investigador a adquirir el artículo y leerlo. Sin embargo, sería necesaria más investigación con la que poder sustanciar este tipo de afirmaciones.

\section{DISCUSIÓN Y CONCLUSIÓN}

Es evidente que el resumen, tanto tradicional como estructurado, "ha de orientarse a definir claramente lo que el artículo trata" (Day, 2005: 28) y los resultados obtenidos nos indican que seguir una pauta estructural es una garantía de que el resumen contenga la información más adecuada posible. Es asimismo evidente que la Medicina en cierto modo ha arrastrado una buena parte de los profesionales de Enfermería a publicar sus artículos con resúmenes estructurados, aunque quizás excesivamente diversificados. Piqué-Angordans et al. (2009) presentaron evidencia significativa sobre las veinte primeras revistas españolas de Enfermería, según el ranking CUIDEN (índice de repercusión histórica $\left.\mathrm{Rc}_{\mathrm{h}}\right)^{3}: 18$ de ellas $(90 \%)$ publican según la normativa médica 
Vancouver, una según el modelo APA, y otra admite artículos según ambos modelos. En cambio, se observa una tendencia al alza del modelo APA en las 36 revistas punteras anglosajonas (según el Science Citation Index de 2006): 16 revistas publican según el modelo Vancouver, mientras que 20 de ellas $(55,56 \%)$ siguen el modelo APA.

Como se ha dicho anteriormente:

"los tiempos en que la Enfermería era simplemente una destreza, una formación profesional sofisticada o incluso un apéndice de la medicina, han pasado a la historia" (Piqué-Angordans et al., 2011: 13).

La Enfermería debe tener su propio estilo y modo de publicar ya que no todo lo que se publica en esta disciplina debe encasillarse en los modelos biomédicos de investigación. Los comités de redacción de las revistas de Enfermería deben también entender que un resumen bien estructurado es un elemento de venta del artículo, una herramienta de marketing en definitiva, que contribuye a una mejor difusión de la investigación de los profesionales a través de las bases de datos internacionales, pero también al prestigio de su vehículo, es decir, la revista.

En este estudio se han observado carencias que pueden ser subsanadas a la hora de confeccionar los resúmenes cuya mejora puede generar un mejor funcionamiento del resumen como reclamo. La variabilidad observada en el conjunto de 240 resúmenes de Enfermería estudiados, a menudo con una falta evidente de información (más del $65 \%$ de ellos solo contienen entre una y tres unidades estructurales, y solo el $35 \%$ de los resúmenes contienen 4 ó 5 unidades), debe servir de acicate a la hora de preparar un artículo para su publicación. En la actualidad, las revistas ya no suelen disponer de un equipo de redactores de resúmenes, sino que la mayoría solicita que sea el propio autor quien lo redacte. Es por ello que merece la pena dedicarle el tiempo necesario para su redacción y seguir unas pautas que se centran básicamente en incluir una información necesaria para que sea el elemento decisivo en lo que Hyland (2000) llamaba la marketisation del artículo.

Ante la diversidad de tipos de publicaciones que aparecen en las revistas de Enfermería, sus comités de redacción deben, por lo tanto, plantearse especificar con mayor claridad las directrices de publicación en las mismas. No se trata de rechazar el resumen tradicional de un párrafo o el resumen estructurado, ya que ambos pueden cumplir perfectamente su misión, sino de unificar criterios y proponer una estructura suficientemente simple aplicable a la Enfermería en general en la que tengan cabida tanto los artículos basados en datos empíricos, como los artículos que informan sobre casos concretos, que ofrecen análisis e información sobre técnicas de Enfermería, o artículos como el presente, en el que se analizan textos. Lo importante, en definitiva, es conseguir que el autor sepa 'vender' su producto mediante un resumen convincente para que los lectores lean el artículo. 


\section{REFERENCIAS BIBLIOGRÁFICAS}

Ad Hoc Working Group for Critical Appraisal of the Medical Literature (1987). A proposal for more informative abstracts of clinical articles. Annals of Internal Medicine, 106, 598-604.

ANSI/NISO (1997). Guidelines for abstracts. Revision of ANSI Z39.14-1979 (R1987). Bethesda, MD: American National Standards Institute, NISO Press.

APA (2010). Publication Manual of the American Psychological Association. Washington, D.C.: American Psychological Association.

Bazerman, C. (1988). Shaping written knowledge: The genre and activity of the experimental article in science. Madison: The University of Wisconsin Press.

Cross, C. \& Oppenheim, C. (2005). A genre analysis of scientific abstracts. Journal of Documentation, 62(4), 428-446.

Day, R. (2005). Cómo escribiry publicar trabajos cientificos. Washington, DC: Organización Panamericana de la Salud.

Díez, M. B. (2007). El resumen de un artículo científico. Qué es y que no es. Investigación y Educación en Enfermería. 25(1), 4-17.

Fernández Martínez, A. V., Soto Ureña, S., Arenas Fuentes, M., Horrillo Jiménez, F., Sáez Donaire \& Pérez Valencia, L. (2010). Comparación de infusión automática respecto a manual en hemodiafiltración 'on line' post-dilucional. Enfermería Nefrológica, 13(1), 17-22.

Fortanet Gómez, I. (Coord.) (2002). Cómo escribir un artículo de investigación en inglés. Madrid: Alianza Editorial.

Gálvez Toro, A., Hueso Montoro, C. \& Amezcua, M. (2004). Indicadores CUIDEN de repercusión de las revistas de enfermería del área lingüística del español y del portugués. Index de Enfermería, 13(46), 76-80.

Hartley, J. (1997). Is it appropriate to use structured abstracts in social science journals? Learned Publishing, 10, 313-317.

Hartley, J. (2002). Do structured abstracts take more space? And does it matter? Journal of Information Science, 28, 417-422.

Hartley, J. \& Betts, L. (2009). Common weaknesses in traditional abstracts in the Social Sciences. Journal of the American Society for Information Science and Technology 60(10), 2010-2018. 
Hyland, K. (2000). Disciplinary discourses. Social interactions in Academic Writing. Harlow: Longman.

Kandzior, A. (2009). Ambiente de influenza, en la espera de un Centro de Salud Familiar. Enfermería Comunitaria [en línea]. Disponible en: www.index-f.com/ comunitaria/revista.php

Martín Martín, P. (2003). Análisis contrastivo de los componentes estructurales y gramaticales de los resúmenes de los artículos científicos. Revista Española de Lingüistica, 33(1), 153-183.

Martín Martín, P. (2010). El inglés para fines académicos: Aportaciones en la investigación y enseñanza del discurso científico. Revista de Lingüistica y Lenguas Aplicadas, 5, 109-121.

Mosteller, F., Nave, B. \& Miech, E. J. (2004). Why we need a structured abstract in Education Research. Educational Researcher, 33(1), 29-34.

Nakayama, T., Hirai, N., Yamazaki, S. \& Naito, M. (2005). Adoption of structured abstracts by general medical journals and format for a structured abstract. Journal of the Medical Library Association, 93(2), 237-242.

Newell, R. (2000). Writing academic papers: The Clinical Effectiveness in Nursing experience. Clinical Effectiveness in Nursing, 4, 93-98.

Otañi, I. (2005). El resumen o abstract del artículo de investigación en español: Características retórico-lingüísticas. En G. Vázquez (Coord.), Español con fines académicos: De la comprensión a la producción de textos (pp. 45-72). Madrid: Editorial Edinumen.

Perea Baena, J. M. (2008). Formación en estrategias de mejora para Auxiliares de Enfermería. Un curso pertinente. Enfermería Docente [en línea]. Disponible en: www.index-f.com/edocente/revista.php

Pho, P. D. (2008). Research article abstracts in applied linguistics and educational technology: A study of linguistic realizations of rhetorical structure and authorial stance. Discourse Studies, 10(2), 231-250.

Piqué-Angordans, J., Camaño-Puig, R. \& Piqué-Noguera, C. (2009). La investigación en enfermería y los modelos de citación bibliográfica. Revista Latino-americana de Enfermagem [en línea]. Disponible en: http://ead.eerp.usp.br/rlae/

Piqué-Angordans, J., Camaño-Puig, R. \& Piqué-Noguera, C. (2011). Escribir y publicar en Enfermería: Del trabajo escrito universitario al articulo de investigación. Valencia: Tirant lo Blanch Humanidades. 
Piqué-Noguera, C. (2012). Writing business research article abstracts: A genre approach. Ibérica, 24, 211-232.

Posteguillo Gómez, S. (2002). El Título y el Resumen de un artículo de investigación. En I. Fortanet Gómez (Coord.), Cómo escribir un artículo de investigación en inglés (pp. 32-55). Madrid. Alianza Editorial.

Posteguillo Gómez, S. \& Piqué-Angordans, J. (2007). El lenguaje de las ciencias médicas: Comunicación escrita. En E. Alcaraz Varó, J. M. Mateo Martínez \& F. Yus Ramos (Eds.), Las lenguas profesionales y académicas (pp. 167-178). Barcelona: Ariel.

Rodríguez Borrego, M. A., Galván Ledesma, J., Medina Valverde, M. J., de la Torre Barbero, M. J., Romero Bravo, A. \& Flórez Almonacid, C. I. (2010). Evolución del diagnóstico enfermero en el Hospital Universitario Reina Sofía de Córdoba (2005-2009). Evidentia [en línea]. Disponible en: www.index-f. com/evidentia/inicio.php

Rodríguez Martín, M. (2009). La soledad del anciano. Gerokomos [en línea]. Disponible en: http://dialnet.unirioja.es/servlet/revista?codigo $=2433$

Salager-Meyer, F. (1990). Discoursal flaws in medical English abstracts: A genre analysis per research-and text-type, Text, 10(4), 365-384.

Scott, M. (2009). WordSmith Tools. Oxford: Oxford University Press.

Shapin, S. (1984). Pump and circumstance: Robert Boyle's literary technology. Social Studies of Science, 13, 481-520.

Swales, J. M. (1990). Genre Analysis. English in Academic and Research Settings. Cambridge: Cambridge University Press.

Swales, J. M. \& Feak, C. B. (2009). Abstracts and the writing of abstracts. Ann Arbor: The University of Michigan Press.

Vizmanos Sevilla, A. C., Alonso Ugartemendía, M. A., Arenas Olmo, A., Montilla Martín, A. J., Ruiz Serrato, R. \& Grupo de Cuidados Obstétricos (2010). Relación entre cambios posturales e hipotensión en mujeres parturientas con analgesia epidural. Resultados preliminares. Enfermería Docente, 91, 10-15.

Weissberg, R. \& Buker, S. (1990). Writing up research. Experimental research report writing for students of English. Englewood Cliffs, NJ: Prentice Hall Regents. 


\section{NOTAS}

1 IMRD, por Introducción, Material y Método, Resultados y Discusión/Conclusión.

2 En el momento de iniciar esta investigación todavía no disponíamos de acceso a los artículos de 'Enfuro' publicados en 2011.

3 Sobre el índice de repercusión histórica Rch, véase Gálvez Toro, Hueso Montoro y Amezcua (2004). 